\title{
TRANSMER, hydrodynamic model for medium- and long-term simulation of radionuclides transfers in the English Channel and southern North Sea
}

\author{
P. Bailly du Bois ${ }^{1}$ and F. Dumas ${ }^{2}$ \\ ${ }^{1}$ Institut de Radioprotection et de Sûreté Nucléaire, Laboratoire de Radioécologie \\ de Cherbourg-Octeville (IRSN/DEI/SECRE/LRC), \\ rue Max Pol Fouchet, BP. 10, 50130 Cherbourg-Octeville, France \\ ${ }^{2}$ IFREMER/DEL Applications Opérationnelles, \\ Centre de Brest, Z.I. de la Pointe du Diable, BP. 70, 29280 Plouzané, France
}

\begin{abstract}
TRANSMER hydrodynamic model simulates dispersion of dissolved substances in seawater at the scale of the English Channel and the south of the North Sea with a mesh size of one kilometre and a time step of 30 minutes. This model evaluates the medium- and long-term consequences of releases of soluble pollutants in the marine ecosystem under normal or accidental conditions, at a very low computation cost (it takes less than one hour to simulate one year). It has been qualitatively and quantitatively validated by 1400 measurements of ${ }^{125} \mathrm{Sb}$ in seawater in the English Channel and the North Sea. Budgets of measured and simulated radionuclide quantities are balanced for theses seas. The correlation factor between individual measurements in seawater and calculation results is 0.88 with an average error of $\pm 54 \%$, the error attributable to the measurement process being $15 \%$ on average. TRANSMER appears as a robust tool for modelling medium- and long-term consequences of releases of soluble pollutants in the marine ecosystem under normal or accidental conditions.
\end{abstract}

\section{INTRODUCTION}

The assessment of the impact of polluting substance releases into a marine environment under either normal or accidental conditions is an essential issue in coastal development. Hydrodynamic models can simulate the dispersion of soluble substances with very variable geographical areas and calculation resolutions. The "Lagrangian barycentric" method perfected by Salomon and Orbi [1][2], has made it possible to calculate the displacement and dilution of chemical substances in solution in seawater over large time scales (several years) and large space scales (several thousand kilometres). One of the major appeals of such models is the direct comparisons between soluble substance concentrations obtained from simulations and the results of in-situ measurements, the field data generally resulting from the transport of the substances in the seawater over distances of several tens or hundreds of kilometres, for weeks and months.

These models require field data of similar range and accuracy to be validated. The measurements of radiotracers released by the nuclear fuel reprocessing plants are outstanding opportunities for such validations. The general distribution of the water masses from the Channel and the North Sea have thus been specified by measurements of soluble radionuclides, the general diagram of these movements is shown in Figure 1 [8].

The antimony 125 released from the COGEMA-La Hague nuclear fuel reprocessing plant is particularly interesting with regard to water circulation in the Channel and the south of the North Sea: it is the principal source of this radionuclide in this region and has been known accurately, release by 
release, since 1982; the radioactive period of ${ }^{125} \mathrm{Sb}$ is long enough (2.7 years); very low concentrations of seawater can be measured (concentrations of $0.3 \mathrm{~Bq} \cdot \mathrm{m}^{-3}, 40,000$ times lower that the natural radioactivity of seawater); the field measurements database available for the validation is particularly large.

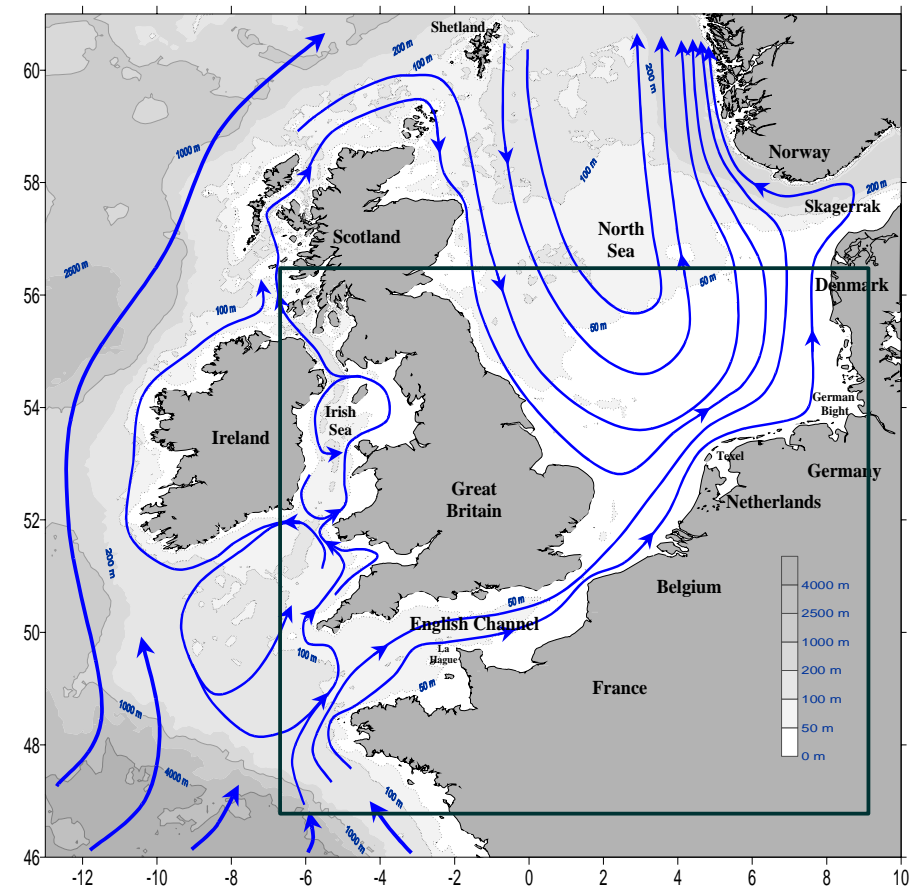

Figure 1. General circulation of water masses in the seas of North-western Europe [8], and frame of the residual model.

The results obtained with the ${ }^{125} \mathrm{Sb}$ and a model of residual currents during previous work have overall agreed clearly with the calculated and measured results. The average distribution of the radionuclides measured and observed has been reproduced at the scale of the Channel and the North Sea [4] [5] [6]. However, a significant discrepancy has appeared between the assessments of calculated and measured radionuclides and in the spatial spread of the plumes marked at a given date. This study specifies the constraint parameters of the hydrodynamic model, particularly the meteorological forcing, so as to reproduce as faithfully as possible the distributions of soluble tracers observed at the scale of the Channel and the North Sea. The aim is to define a unique set of parameters capable of simulating the dispersion of substances in solution in seawater under realistic wind and tide conditions, at the scale of months and years in the Channel and the North Sea.

\section{RESIDUAL CURRENT MODEL}

The model uses the residual tide currents in barycentric coordinates calculated with a model of instantaneous currents, by applying the method developed between 1985 and 1993 [1] [2]. The detail is given in [1] [2]. This kind of model can reduce calculation times by a factor of 1000 compared with instantaneous current models with similar geographical area and resolution.

Residual current files have been calculated using a model with the same resolution and limits as the residual model, which solves fully hydrodynamic equations for a two-dimensional model (Saint Venant equations). It is a model with spherical coordinates based on the hypothesis that the tide and wind dominate the other mechanisms in the water mass circulations. The mesh resolution is 
around one $\mathrm{km}$ on the entire model frame, which includes one million calculation meshes (Table 1). The limit conditions are provided by a model with a larger frame encompassing the continental shelf of North-western Europe. This model is itself forced by observations of the tidal wave.

Table 1. Hydrodynamic model characteristics required to develop the residual model.

\begin{tabular}{|c|c|c|c|}
\hline & Overall model & Instantaneous current model & Residual current model \\
\hline Geographical limits (frame) & $\begin{array}{c}40^{\circ} \mathrm{N}-65^{\circ} \mathrm{N} \\
20^{\circ} \mathrm{W}-10^{\circ} \mathrm{E}\end{array}$ & $\begin{array}{c}46.82^{\circ} \mathrm{N}-55.98^{\circ} \mathrm{N} \\
6.6^{\circ} \mathrm{W}-9^{\circ} \mathrm{E}\end{array}$ & $\begin{array}{c}46.82^{\circ} \mathrm{N}-55.98^{\circ} \mathrm{N} \\
6.6^{\circ} \mathrm{W}-9^{\circ} \mathrm{E}\end{array}$ \\
\hline Grid & $\begin{array}{c}421 \times 501= \\
210921 \mathrm{mesh}\end{array}$ & $\begin{array}{c}1073 \times 1022 \mathrm{r} \\
1096606 \mathrm{mesh}\end{array}$ & $\begin{array}{c}1073 \times 1022= \\
1096606 \mathrm{mesh}\end{array}$ \\
\hline Mesh size & $\approx 5 \mathrm{~km}$ & $\approx 1 \mathrm{~km}$ & $\approx 1 \mathrm{~km}$ \\
\hline Time step & $240 \mathrm{~s}$ & $20 \mathrm{~s}$ & $\begin{array}{c}\text { Dynamic, } \\
1000 \mathrm{~s} \text { on average }\end{array}$ \\
\hline $\begin{array}{c}\text { Calculation time per day of } \\
\text { simulation }\end{array}$ & $10 \mathrm{mn}$ & $12 \mathrm{~h}$ & $1 \mathrm{~s}$ \\
\hline
\end{tabular}

The $\mathrm{U}$ and $\mathrm{V}$ components were calculated with an instantaneous model using the Lagrangian barycentric method [1] in nine wind situations and for an average tide with a coefficient of 70 . Figure 2 illustrates the structure of the Lagrangian residual current fields for a south-westerly wind of $10.8 \mathrm{~m} . \mathrm{s}^{-1}$. These situations are interpolated according to actual tide and wind conditions when the simulation is performed. As the instantaneous tide currents are not accounted, the model can only be used when the residual currents dominate the instantaneous currents, approximately one week after the start of a release and over $30 \mathrm{~km}$ from the outfall. This calculation method can be used for realistic simulations of radionuclide dispersion over several years.
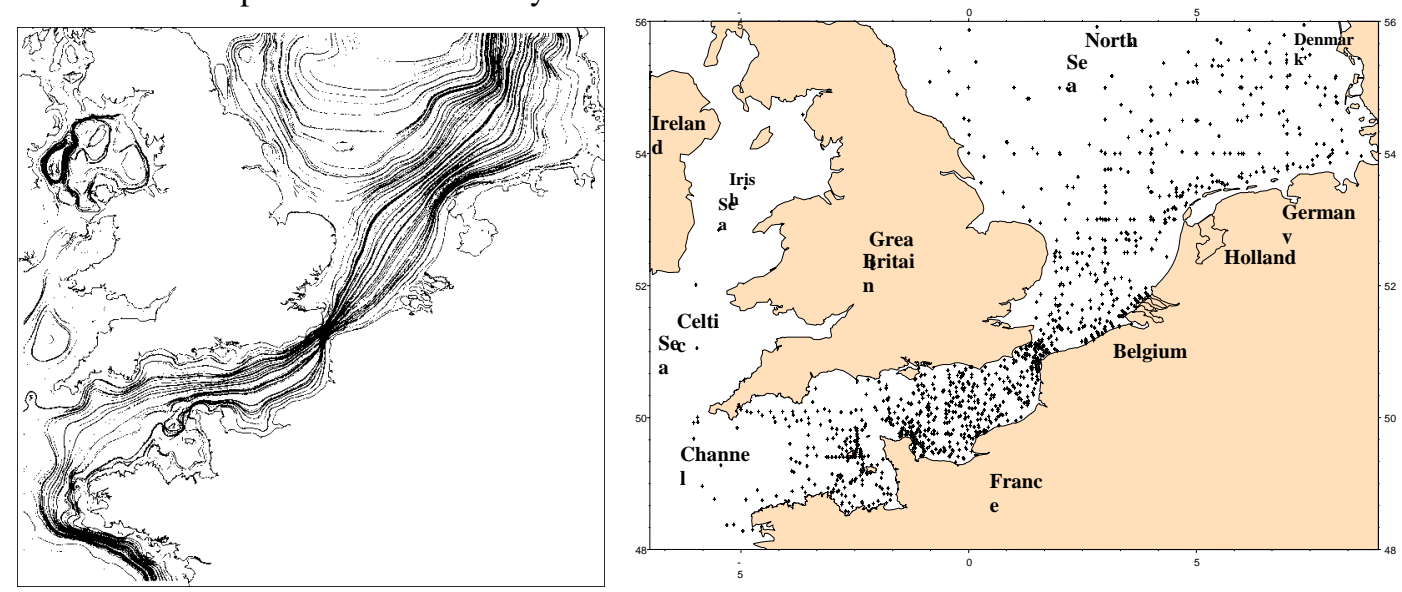

Figure 2. Wind situation in South-west sectorFigure 3. Localisation of 1,400 measurements of $125 \mathrm{Sb}$ ( $\|\vec{U}\|=10.8 \mathrm{~m} / \mathrm{s})$, general view. used to validate the TRANSMER.

\section{DATABASE OF ANTIMONY 125 MEASUREMENTS}

The data used come from IRSN campaigns between 1987 and 1992 on board ships belonging to the French Research Institute for Ocean Exploitation (IFREMER), the National Centre for Scientific Research (CNRS), the Ministry of Agriculture, Fisheries and Food (MAFF, GB) and measurements taken by the French Navy's Atomic Study Group (GEA) and the Institute for Radiological Protection and Nuclear Safety (IRSN-LRC). The majority of measurements presented have already been published and discussed [5][6][7]. The localisations of the samples used for the validation are presented in Figure 3. They cover fully and repetitively the dispersion zone of the release plume from the La Hague plant in the Channel and the North Sea. 


\section{QUANTITATIVE EXPLOITATION OF MEASUREMENTS}

Six sampling campaigns comprised sufficient sampling stations (50 to 156 per campaign) to establish distribution maps of artificial radionuclides released by nuclear industries into the seawater at the scale of the Channel and the North Sea. Measurements at each sampling station were interpolated on the entire field concerned during the sampling campaign by means of regular meshing. The quantities of radionuclides presented during the various campaigns were calculated by multiplying the concentration fields obtained from the interpolation by the bathymetry in each calculation mesh. The model mesh and bathymetry were used for this calculation. The quantities of radionuclides obtained could thus be directly compared with the results obtained from hydrodynamic model simulations.

\section{VALIDATION RESULTS}

Approximately one thousand simulations were performed to validate TRANSMER [8]. The main parameter adjusted was the calculation method for the wind drag coefficient on the sea, which had to be increased considerably. Measured and simulated radionuclide distributions are compared in Figures 4 and 5.

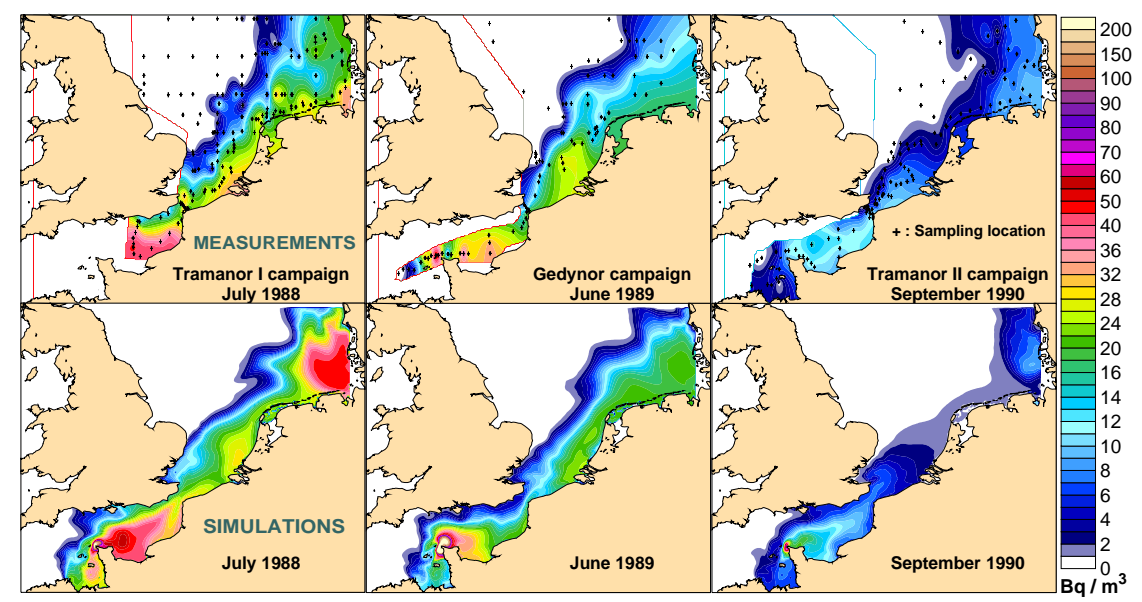

Figure 4. Comparison of measured and simulated antimony 125 distributions in the Channel and the North Sea between 1988 and 1990.

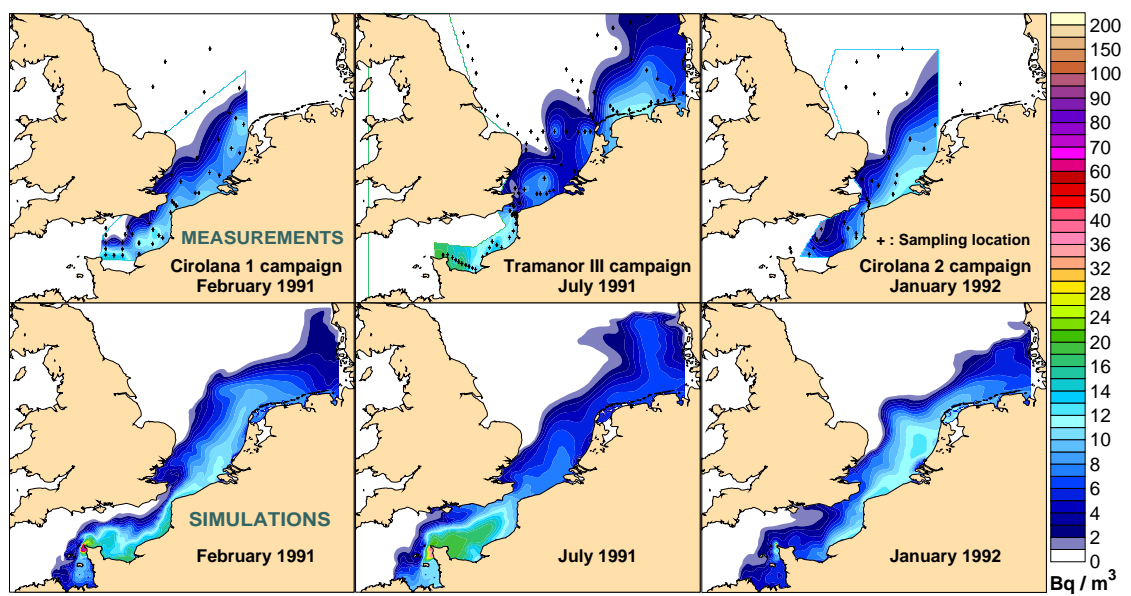

Figure 5. Comparison of measured and simulated antimony 125 distributions in the Channel and the North Sea between 1991 and 1992. 
Using the adopted validation parameters, the relationship between the simulation and measurement results is as follows:

- The average, overall quantities of radionuclides calculated and measured during the six sampling campaigns between 1988 and 1994 are balanced at $\pm 1 \%$, be it over the entire Channel and North Sea dispersion zone or in each sea taken separately;

- The correlation coefficient between the 1,400 measured and calculated concentrations at the same place and date is close to 0.88 ;

- $\quad$ The average discrepancy between the individual calculated and measured values is 54\%;

- For half the 1,400 measurements, the ratio between the measured and calculated concentrations is less than 1.27; this ratio is less than 3.6 for $95 \%$ of the measurements and never exceeds a factor of 12 ;

- The discrepancies between the calculated and measured values are similar at $30 \mathrm{~km}$ or 1,000 $\mathrm{km}$ from the outfall;

- The drag coefficient defined to calculate the frictional force of the wind on the sea is $\mathrm{Cd}=0.0015 \mathrm{x}\|\overrightarrow{\mathrm{W}}\|^{0.48} \mathrm{~m}^{2} \cdot \mathrm{s}^{-1}$ where $\|\overrightarrow{\mathrm{W}}\|$ is the wind module with data from the ECMWF European model;

- A comparison with the most recent measurement results, not taken into account in the set of validation data, shows at least an equivalent agreement with that obtained with data used for the model validation.

Using maps representing the fields of concentration for each campaign, it is possible to calculate the standardised concentration fields by dividing the concentration value in each mesh by the quantity of radionuclides released during the twelve months prior to each campaign. By taking an average of these standardised distributions for all campaigns, it is possible to visualise the average spread of the release plume from the La Hague plant for a given release, in this instance, a constant release of ${ }^{125} \mathrm{Sb}$ of $10^{6} \mathrm{~Bq} . \mathrm{s}^{-1}$. The simulation results were processed in the same way, the maps obtained from measurements or simulations can therefore be compared directly; they are presented in Figure 6.

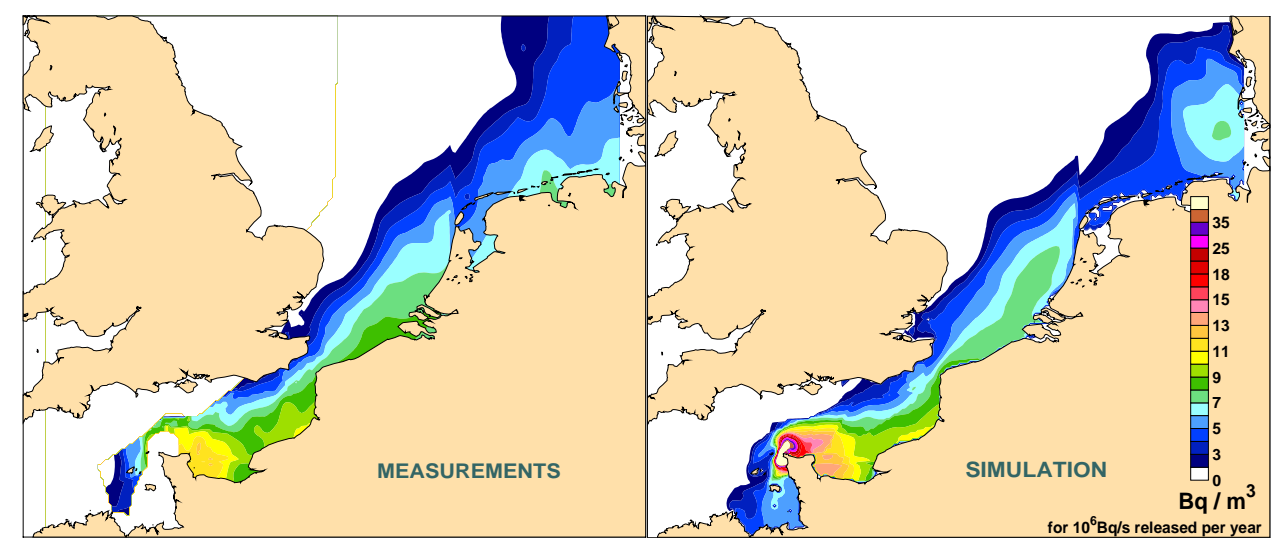

Figure 6. Average measured and simulated antimony 125 distribution between 1988 and 1992, standardised for a release of $10^{6} \mathrm{~Bq} / \mathrm{s}$.

\section{SIMULATION OF TRANSFERS TOWARDS BIOLOGICAL AND SEDIMENTARY COMPARTMENTS}

Calculation modules for transfers towards biological and sedimentary compartments have been incorporated into the hydrodynamic model [9]. These modules simulate the transfers dynamically based on data available in published works. The TRANSMER model can also be used to specify these parameters when field data are available [10]. 


\section{CONCLUSION}

TRANSMER allows the dispersion of chronic or accidental radionuclide releases into a marine environment to be reconstituted or forecast using actual source-terms, winds and tides. It calculates the concentrations in seawater, the living species and sediments on the scale of the Channel and the south of the North Sea. The substances considered by TRANSMER must have soluble behaviour when transported by seawater (density close to that of seawater). The average discrepancy between the 1,400 measured and calculated values is $54 \%$, which is remarkable for concentration simulations in the environment for periods of several years and distances of up to $1,000 \mathrm{~km}$. The hydrodynamic model thus validated forms the basis for an ecological model aimed at reconstituting and forecasting what happens to substances transported in seawater and exchanged with living species and sediments during months and years.

\section{References}

[1] J.C. Salomon, P. Guéguéniat, A. Orbi, Y. Baron, In: Radionucléides: A tool for oceanography. Cherbourg 1-5 juin 1987, Edited by J.C.Guary, P. Guéguéniat, R.J.Pentreath, (Elsevier Applied Science Publishers, 1988), 384-394.

[2] A. Orbi, J.C. Salomon, Océanologica Acta 11, 1 (1988), 55 - 64.

[3] P. Bailly du Bois, F. Dumas, Proceedings of 34th International Liege Colloquium on Ocean Hydrodynamics, Tracer Methods in Geophysical Fluid Dynamics, Liege, 2002 (2002), p. 7.

[4] J.C. Salomon, M. Breton, P. Guéguéniat, Journal of Marine Systems 6, 5-6, (1995), 515-528.

[5] M. Breton, J.C. Salomon, Journal of Marine Systems 6, 5-6, (1995), 495-514.

[6] P. Bailly du Bois, J.C. Salomon, R. Gandon, P. Guéguéniat, Journal of Marine Systems 6, 5-6, (1995), 457-481

[7] P. Bailly du Bois, P. Guéguéniat, Continental Shelf Research 19 (1999), 1977-2002.

[8] P. Bailly du Bois, P. Germain, M. Rozet and L. Solier, In: Proc. Int. Conf. Radioactivity in Environment, Monaco 1 - 5 September 2002, edited by P. Borretzen, T. Jolle and P. Strand, (2002) 395 - 399

[9] P. Bailly du Bois, Rapport IRSN/DPRE/SERNAT/ 2003-04 (2003), 188 p.

[10] B. Fiévet, D. Plet, Journal of Environmental Radioactivity 65 (2002) 91-107. 